\title{
Design and Development of a Low-cost Device for Weight and Center of Gravity Simulation in Virtual Reality
}

\author{
Diego Monteiro ${ }^{*}$ \\ Birmingham City University \\ Birmingham, United Kingdom \\ diego.vilelamonteiro@bcu.ac.uk
}

\author{
Hai-Ning Liang ${ }^{*}$ \\ Xi'an Jiaotong-Liverpool University \\ Suzhou, China \\ haining.liang@xjtlu.edu.cn
}

\author{
Xian Wang \\ Xi' an Jiaotong-Liverpool University \\ Suzhou, China
}

\author{
Wenge $\mathrm{Xu}$ \\ Birmingham City University \\ Birmingham, United Kingdom
}

\author{
Huawei Tu \\ La Trobe University \\ Melbourne, Australia
}
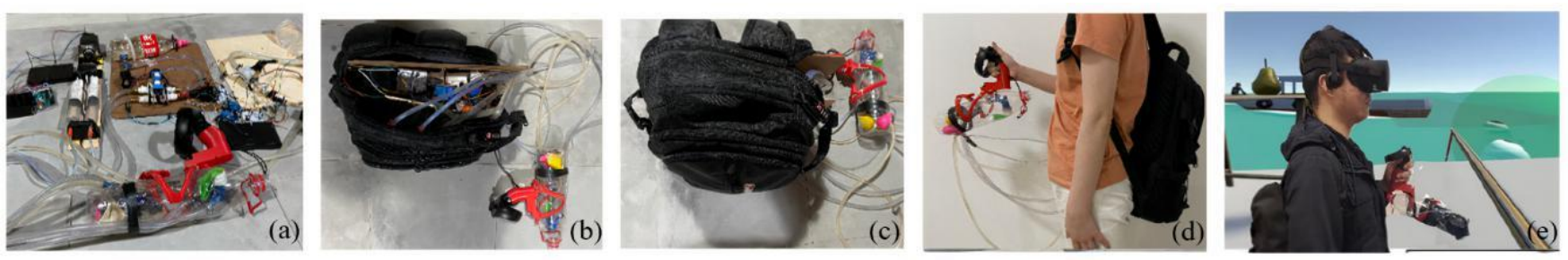

Figure 1: From left to right: (a) all the components of the FluidWeight prototype: 1) syringes, 2) bottles, 3) pumps, 4) threaded road, 5) balloons, 6) 3D printed holder, 7) transmission cables, and 8) Arduino controller; (b) bag open with the parts of the device; (c) FluidWeight as presented to the participants; (d) a user operating the prototype; and (e) another user with the prototype (foreground) and the virtual fishing environment (background).

\section{ABSTRACT}

With rapid advances in virtual reality (VR) technology, the use of haptics has become important to allow users to feel the physical properties of virtual objects. Current research has focused mainly on either weight variation or changing the center of gravity, which limits the simulation potential and may affect the feeling of immersion. This research explores the design and development of a device that can simulate both weight and center of gravity using low-cost components. Through an iterative design process and continuous testing with users, we arrived at a final prototype, FluidWeight, a device that can be attached to a typical VR handheld controller. FluidWeight uses fluid, which is transported from a central storage to a receptacle attached to the controller. A final experiment shows that users enjoyed using it because it could help increase the sense of realism in VR applications.

\section{CCS CONCEPTS}

- Human-centered computing $\rightarrow$ Virtual reality; Haptic de-vices; User studies; $\bullet$ Applied computing $\rightarrow$ Computer games.

\footnotetext{
* Corresponding author
}

\section{KEYWORDS}

Virtual Reality, Force Feedback, Haptics, Weight Distribution, Center of Gravity, Games.

\section{INTRODUCTION}

Sensing an object's weight and how its weight is distributed is one of the main ways people understand objects in real life. However, it remains a significant challenge of immersive virtual reality (VR). In VR, the user who picks up a virtual object with a controller will subconsciously expect changes in the weight of the controller. If an application is to allow a fuller immersive experience, this aspect is of high importance. Most applications and several studies use only vibration or texture as the haptic feedback provided to users $[3,6,19,20,30]$. However, vibration alone cannot reflect suitably all the properties of haptic sensations people feel when holding objects because it cannot completely map other types of information like weight and inertia [19].

People want to engage with virtual environments primarily be-cause of the enhanced feeling of immersion [28], which is crucial for enjoyment and performance, especially in games [13]. Given that haptic sensations are an essential part of the immersive experience in VR [12], it is far from ideal that current commercial VR systems can only provide the same haptic stimuli for all virtual objects. These stimuli are often via limited handheld controllers and can create sensorial conflicts when users interact with objects that have distinct mass properties in the same environment. That is, bulky items (like guns or swords) weigh as little and the same as the controller when they are picked up [30]. Moreover, some objects' weights change during the interaction. For example, a fishing rod should be heavier after catching a fish and a gun lighter after firing all its bullets. However, presenting these changes and 
changing the center of gravity of an object can be challenging to associate in existing VR systems.

Our research intends to close this gap by presenting the design and development process of creating a device that allows participants to feel both the weight and center of gravity of virtual objects [19]. This process led us to try various designs and low-cost com-ponents while at the same time continuously testing to refine the designs with users. The final design, FluidWeight, is a device that can be attached to a typical VR handheld controller, like the Oculus Touch used in our research, and can simulate the weight and center of gravity of virtual objects to provide additional haptic sensations to users (see Figure 1). We report the user experiments and design choices that were made in the process of building this device that, unlike previous technologies, has the following five main features: (1) it can change its mass $[19,21,22,25,27,30] ;(2)$ it is relatively silent and fast $[7,10]$; (3) it can change its center of gravity in 1D [2, 16]; (4) its materials are cheap and easily accessible [16]; and (5) it can be integrated with current VR technologies, such as the Oculus. In short, the main contribution of this research is a device that bring these five features together. In addition, our user studies conducted in this iterative process can give practical insight into the human and technical factors that are important to consider building a haptic system that simulates weight and is compatible with current HMDs. These lessons and insights can be used to frame the design of other similar devices.

This paper is organized as follows. The following section presents research related to our work, focusing on techniques for weight simulation. Then, we describe the iterative development steps in the development of FluidWeight. In this part, we provide the three variations of our device and show how it can enhance the sense of immersion through an incremental and iterative process and their corresponding studies. In each part, we present the design used and explain why and how we altered the previous design. We then present the results of a final experiment and the conclusion of the paper.

\section{RELATED WORK}

There are two main types of weight simulation, pseudo-haptic feedback, and mechanical force. After a summary of the literature on pseudo-haptic feedback and, because FluidWeight is a mechanical force device, we focus especially on it and its principles that have influenced our design choices.

\subsection{Pseudo-haptics}

Paul et al. [8] proposed that users could intuitively identify heavy objects by using visual cues. Lionel et al. [4] concluded experimentally that the control/display ratio impacts the perception of the mass of virtual objects. Control/display ratios below 1 create the illusion of lighter virtual objects, but the opposite is found with values above 1 [29]. Applying this method, David et al. [9] success-fully used a Kinect to artificially modify the avatars' animation giving their participants access to different weights of dumbbells. Despite their positive affordances, pseudo-haptic approaches have their drawbacks. It is difficult to simulate larger loads [11], can only be used primarily to determine relative values [29], and cannot be used without visual cues.

\subsection{Mechanical Feedback}

Mechanical force uses physical devices to impose force on the hu-man body, thereby allowing users to feel the weight of a virtual object. It is fundamentally divided into Active Haptic Feedback and Passive Haptic Feedback [30]. Active Haptic Feedback uses computer-controlled actuators to apply force to VR users to help them experience tactile and kinesthetic stimuli [30]. Passive Hap-tic Feedback does not use any computercontrolled actuators but instead associates the forces generated in the VE with forces from the real world [30]. As such, Passive Haptic Feedback is limited by the physical structure of the real world rather than the VE.

Active Haptic Feedback can have different kinds of actuators generating force. For example, AirGlove [5], Thor's Hammer [7], and AeroPlane [10] use air to simulate forces up to 100 grams on the hand. Gravity Grabber [15] and Grabity [3] use some skin deformation to create the feeling of holding an object. However, relying on rollers to create the illusion of touch is limited because the feeling is restricted to that region only. A significant limitation of some Active Haptic Feedback devices is the noise they emit, which is not conducive to creating a strong sense of immersion in the VE [3]. Further common limitations involve their complexity, price, and user safety [14, 22, 25, 27, 30].

On the other hand, a common dilemma faced by most Passive Haptic Feedback-based devices is poor adaptability to different environments and scenarios [1, 30]. One example is the Elastic-arm [1], an elastic armature installed on the body. When the user's arm is extended, users would feel the self-centered progressive force brought by the Elastic-arm device and feel a series of forces such as the weight in the VE.

Since Active and Passive Haptic Feedback devices individually present drawbacks, Zenner et al. [30] proposed a hybrid device named Shifty, which shifts weight. Like an Active Haptic Feedback device, Shifty can be controlled by a computer to move the center of gravity to change its passive tactile characteristics in 1-Dimension (1D). Their experiments proved that the hybrid device was more realistic and enjoyable than Passive Haptic Feedback [30]. However, the device needs quite a long time (128 seconds) to adjust, which is unsuitable to maintain a continuous sense of immersion in real-time [30]. Another hybrid device, Transcalibur [19], can change its center of gravity in 2D; however, like Shifty, it cannot change its overall weight either.

Other hybrid devices involve the use of air (PuPoP [24]) to change the form and water (GravityCup [2]) to adjust the weight of virtual objects. GravityCup does not alter the center of gravity and can only simulate containers. Similarly, in [16], a weight-changing system is proposed using liquid metal pumped into the grabbed object to achieve weight simulation. However, their components and materials used are expensive, and, more importantly, their design cannot alter the center of gravity.

In sum, providing realistic haptic feedback is challenging but often improves interaction and the VR experience [6, 21, 26]. Our fi-nal prototype, FluidWeight, is also a hybrid feedback device and can provide realistic weight sensations and changes in the objects' cen-ter of gravity through fast fluid input-output control. It is portable, fast, and based on low-cost components and allows greater simulation complexity than other fluid-based devices (like GravityCup [2]).

\section{SYSTEM IMPLEMENTATION}

We focused on a portable device so that users could leverage the properties of VR and be able to stand and move freely. We explored weight redistribution to allow mobility. The mass should be relocated from an unperceivable reservoir to the desired place (e.g., the hands). A backpack was used to contain the reservoir because its position on the users' back gives centrality and allows for a natural weight (re)distribution. 


\subsection{Design Requirements}

We identified a set of requirements for the system: (1) it should be able to simulate small everyday objects (e.g., a small hammer or a mug with water weighing $0.6 \mathrm{~kg}$ ); (2) it should be reasonably silent and offer a fast response so that the weight difference should be felt almost instantly when the user grabs a virtual object without drawing attention to the change; and (3) it should be able to integrate itself with current VR technologies, such as the Oculus Rift.

\subsection{Simulating Mass}

In our implementation, we employed water to provide the weight after excluding other options. The reasons for their exclusion were (1) liquid metals can be toxic or difficult/expensive to acquire [16]; (2) oils have low density and would require greater volumes to achieve the desired effect [17], they are often more expensive than water as well; (3) sugar-based mixes have high viscosity [23] and would make the prototype slow or require heavy components, which are unsuitable for a portable, lightweight device. We chose water as it is cheap, has a density of about $1 \mathrm{~g} / \mathrm{cm} 3$, and has been used in previous research [2]. A closed-circuit system was required to keep the content from spilling. However, if the receptacle walls were solid, the pressure could either break them or increase the system's energy demands. After evaluating several options, we opted to use party balloons because they are light, easily expandable, cheap, readily available, and durable under our test conditions. While suitable to contain fluid, balloons can cause a wobbly sensation, which we perceive as limiting. To address this, we experimented with ways to hold the balloon. We chose a plastic bottle to contain the balloons inside them. The bottle is rigid and light, and the balloons fit well enough within it to keep the consistency under various conditions. In total, the receptacle region weighed $220 \mathrm{~g}$ without the controller that weighted $160 \mathrm{~g}$ with batteries.

\subsection{Systems Control}

The Microcontroller logic was the same for all our designs and was based on activation time and direction. After calibration, the microcontroller saved the state of each receptacle. All communication was serial, which did not present any lag in our context. The formulas for calculating how much time each actuator had to be on were pre-programmed into the microcontroller.

Table 1: Symbols used in the formulas

\begin{tabular}{cl|cl}
\hline Symbol & Meaning & Symbol & Meaning \\
\hline$V$ & Volume & $L$ & Shaft rotation distance \\
$m$ & Mass & $n$ & Number of steps \\
$\rho$ & Density & $d$ & Diameter of the shaft \\
$h$ & Height or plunger distance & $m 1$ & Subdivision of stepper motor \\
$r$ & Plunger radius & $C$ & Constant derived from other factors \\
\hline
\end{tabular}

We followed a series of well-known formulas to derive ours (see Table1 for the meaning of the symbols). First, we established the formula for the volume (1) and the volume of the syringe that is used to inject water (2). From this, we could calculate the plunger distance (3), which was equal to the distance moved by the shaft given a certain number of steps (4). To calculate the number of steps, we combined (3) and (4) into (5). We based our code on three assumptions: (a) the same electric power spun the motor at the same rate; (b) only the plunger's position affected the variance in volume; and (c) the density of the material was constant. So, the simplified formula (6) only requires changes in mass.

$$
\begin{gathered}
V=\frac{m}{\rho} \\
V=h \pi r^{2} \\
h=\frac{V}{\pi r^{2}} \\
h=L=\pi n\left(\frac{d}{360}\right)\left(\frac{1.8}{m 1}\right) \\
n=\frac{360 h m 1}{1.8 d} \therefore \frac{200 m 1 m}{d \pi \rho r^{2}} \\
n=200 \frac{\mathrm{m}}{\mathrm{C}}
\end{gathered}
$$

\section{FLOW SYSTEMS AND USER EXPERIMENTS}

When holding a virtual object, the controller of the weight simulator would activate the transport system of the liquid, moving it into the desired receptacles. We started with a thread rod method to control two reservoirs (syringes). For the initial analysis, we used the value of $15 \mathrm{~g} / \mathrm{s}$ as an acceptable speed. We reevaluated this value through user studies and redesigned the flow system as we obtained further insights from participants' feedback.

There was a total of five incremental user studies, which helped us to verify: (1) the just noticeable weight the system could simu-late; (2) how different initial values affected the minimal perceivable weight; (3) if the system could successfully simulate different cen-ters of gravity; (4) if the system could accurately represent virtual objects; and (5) the effectiveness and usability of integrating Fluid-Weight into a VR application. All experiments lasted fewer than 10 minutes and were one week apart to avoid fatigue.

The same 10 participants ( 6 males, 4 females) participated in all experiments to provide continuity and consistency for data gathering. They were recruited from a local university. Three were left-handed, while the other seven were right-handed. Their age ranged from 20 to 27 years old, with a mean age of 23.2. None declared a history of physical or muscular discomfort. They all had some level of experience with VR and consented to participate in the experiments voluntarily.

\subsection{Threaded Rod}

Our design used a threaded rod approach that can move bigger gauges of individual syringes $(100 \mathrm{ml})$. Each syringe would move at a rate $(12.5$ $\mathrm{ml} / \mathrm{s}$ ). This was a linear actuator in which the force was performed closer to the motor, and as such, it could move large loads (greater mechanical advantage). On the other hand, each rotation was slow because the distance moved by the actuator would be small. This was not a concern because more than one syringe could be moved at once, and the container effectively resulted in $25 \mathrm{ml} / \mathrm{s}$.

\subsection{Experiment A: Absolute Threshold}

The first experiment aimed to detect the Absolute Threshold a user could feel when holding the controller. We used the following pro-cedure to identify users' perception of this threshold. We positioned the balloon at the center of the bottle. Then, we activated the system until the participant declared having felt a change in weight. 
We asked the participants in our experiment to sit and to position their elbow on the table (like they were about to arm-wrestle) to mitigate any effects of fatigue. This method was repeated for all experiments, except in the last experiment, which tested the full device with an VR application. At the beginning of the experiment, each participant was requested to say "OK" when ready to start and to say "Yes" or "I felt it" when they felt a change in weight. For each turn, we took the controller back from the participant and reset the water volume to the baseline. We recorded the corresponding amount of water after the participants' response. We repeated this process three times. On average, each time lasted about 6 seconds with 30 -second intervals to reduce the impact of previous turns.

4.2.1 Results and Discussion. The average of the dominant hand was $22.5 \mathrm{~g}$ ( tandard error (s.e.) $=9.88 \mathrm{~g}$ ) and $19.9 \mathrm{~g}$ (s.e. $=9.83 \mathrm{~g}$ ) for the weak hand. The mode, when rounded to the closest multiple of 5 , was $20 \mathrm{~g}$ for the weak hand and $25 \mathrm{~g}$ for the dominant hand. These results suggest that for equipment attached to the controller, changes should focus on incremental values of at least $20 \mathrm{~g}$ because finer variances would probably go unnoticed. The results indicate that the pump can be an adequate solution and that the subsequent studies can be performed discretely.

\subsection{Experiment B: Center of Gravity Detection Accuracy}

We performed this study to identify if participants could detect different centers of gravity. For each trial, participants reported which balloons had been filled, if any, and would point at the chart shown in Figure 2. There were four conditions: all Empty balloons, filled Front balloon, filled Back balloon, and Both balloons half-filled.

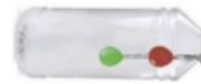

(a) (b)

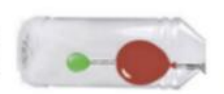

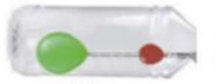

(c)

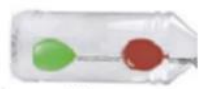

(d)
Figure 2: Four configurations of the center of gravity: (a) no balloon is filled (Empty); (b) the back balloon is filled (Back); (c) the front balloon is filled (Front); (d) both balloons are half-filled (Both). The summed weight is $50 \mathrm{~g}$.

We asked participants to look away from their arms to avoid getting any visual cues. The experiment always started with the participants holding the empty device to build a baseline and then releasing it for a 5second filling. They then received the device and had to point to a condition that reflected the current state of the device. The process was repeated four times. The balloons' combined summed weight was $50 \mathrm{~g}$ after being filled. Even though participants only received each condition once, they were unaware of how many times the procedure would be repeated. Thus, they could choose the same condition more than once if they thought it was the correct one. We based the weights on a lever system, the position of the balloons, and the results from Experiment A.

4.3.1 Results and Discussion. To analyze the collected data, we did a graphical analysis of (1) what the real position was against (2) what the participants believed it to be (see Figure 3). The results revealed that, overall, participants could distinguish well between Empty and the other conditions. This further confirms the positive results from the previous experiments, with the weight threshold of $50 \mathrm{~g}$ being a good baseline. Because the two most common confusions are between (1) Back and Empty and (2) Both and Front, it shows that the front balloon was the most influential in the weight sensation. This result is aligned with the concept of levers, given that the hand is the fulcrum and the central part of the system.

Likely, participants who were first exposed to the Front balloon believed intuitively that two balloons would be more massive. A participant who was less sensitive to changes would likely not see a difference when the center of gravity was closest to their hands (Back) and when there was no change (Empty). Given that most conditions were accurately detected, it confirmed that the current model could be used for our subsequent investigations. Furthermore, a combination of both balloons should be explored when the goal is to expedite the weight variation. However, the highest caliber of syringe this version could transport adequately was $50 \mathrm{ml}$. As such, even though the system can control one syringe, to move large amounts of liquid, it was necessary to build an array of motors, but this could make the system bulky and require a larger space. Thus, we must move on to a new design.

\subsection{Experiment C: Rate and Clarity of Change}

In the previous experiment (session 4.2), participants felt the changes discretely. In this experiment, we explored the time to fill the receptacles as perceived by participants. Based on the previous experiment, we only had two conditions (A) one balloon in the front (Figure 2c), or (B) two distributed balloons being equally filled (Figure 2d).

The balloon in condition A was filled in 4 seconds, while both in condition B were filled in 2 seconds. For each condition, the balloons started empty (dry mass weight of $380 \mathrm{~g}$ ). Participants were then asked on a 3-point Likert scale two questions: (1) how clear the change had been, and (2) how fast the change had been. The 3-point Likert scale choices were (1) "Slow," "Acceptable," and "Fast" in terms of speed change; and (2) "No Change," "Slight Change," and "Clear Change" in terms of how perceivable the change was. The participants were instructed to choose (1) "Slow" if the wait for the change was taking longer than they expected; (2) "Fast" if they felt the change in mass happened before they paid attention to the increments; and (3) "Acceptable" if it was something in between. "Clear Change" represented a difference in mass that could be easily perceived; that is, they did not feel they needed to pay attention to feel the change. The participants were asked to try to separate the rate of change from the speed.

\section{Real Position vs Perceived Position}

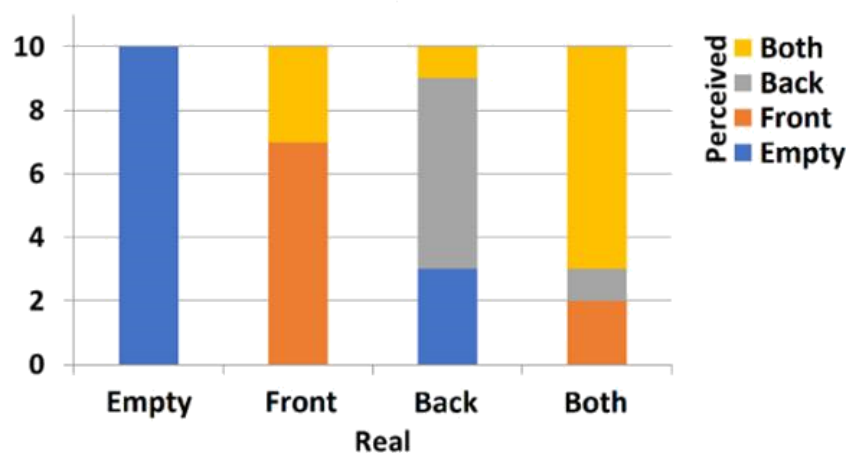

Figure 3: Confusion graphs showing a comparison between what center of gravity was presented to the participants against what they believed to be the case. 
4.4.1 Results and Discussion. $80 \%$ of participants felt that condition B was Fast, while $20 \%$ felt that it was Acceptable. In contrast, only $60 \%$ of the participants categorized the change as fast in condition A. However, the change was the clearest when participants experienced condition $\mathrm{A}$, in which $70 \%$ felt an evident change. Most participants (80\%) felt only a slight change. Interestingly, one participant declared not having felt any change but declared that the speed was acceptable. Through these metrics, neither condition is adequate to continue the study; one being perceptible but too slow and the other being fast but virtually imperceptible.

4.4.2 Redesign, Results, and Discussion. We decided to do a re-design. We used individual pumps for filling and emptying the back balloon. Figure 4 shows the pump system. There were four main parts to this system: (1) the fluid reservoir; (2) pumps; (3) the solenoid valve; and (4) the receptacle and fluid transmission system. One of our concerns was to make the system compatible with a backpack. As such, this design had the reservoir positioned on the top. Since regular VR use occurs with the users standing straight or sitting, the fluid would then be able to flow naturally from the reservoir to the hose pipe. When the user held a virtual object, one pump would push the water into the receptacle and, when the user releases the object, the other pump would pull water out. The nor-mally closed solenoids were energized simultaneously with their respective pumps, effectively creating two separate transmission systems.

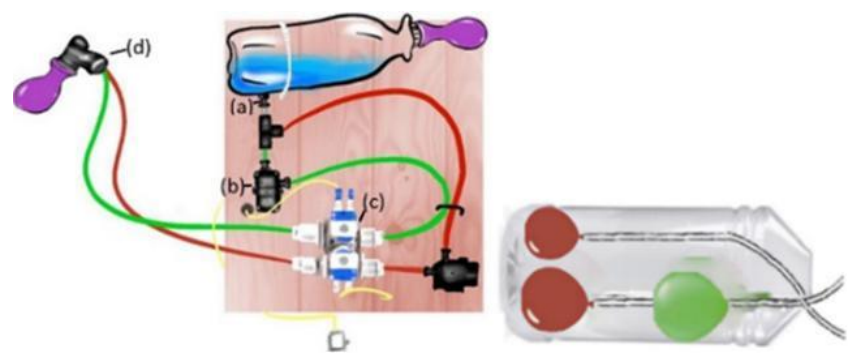

Figure 4: (Left) Pump-based weight simulation system: (a) bottle interface; (b) pump; (c) solenoid valve; and (d) holder. The green and red lines represent the forward and return channels. (Right) The final receptacle system: two balloons in the front and one in the back. Note: Figures 7 and 8 in the appendix at the end of the paper show more details about the components of the system and the 3D printed unit that allowed connecting the receptacle containing the balloons to the VR handheld controller.

This system was fast compared to the other two designs, capable of moving the fluid at speeds of up to $66 \mathrm{ml} / \mathrm{s}$. Further, there was not a definite limit to the size of the reservoir (which was set at $500 \mathrm{ml}$ ). Finally, the error was acceptable and would not impact our applications as already analyzed earlier.

The final design was developed to improve the clarity for users to feel the weight change. It added a third balloon in the front position (see Figure 4). We used the threaded rod method to control the front balloons and associated them with the pump system in the back. The balloons required finer adjustment in the front and fast in the back. After redoing the experiment, all participants could clearly feel the changes in weight. Moreover, $90 \%$ of the participants felt that the rate of change was fast, and they were still able to sense the changes clearly. As these results suggest that this new design was suitable, we used it in the final experiments.

\subsection{Experiment D: Virtual Object Detection Accuracy}

Given the results from Experiments A and B (Sessions 4.2 and 4.3) showed that 30-gram weight increments were perceivable and changes in the center of gravity were easily noticeable with the new design (Experiment D), the purpose of this experiment was to test how accurately participants could distinguish the virtual objects based on their perceived weight.

We first empirically tested the weight of real-life objects using a standard scale and separating the objects into parts and weighting these parts as possible (see Figure 5). We then designed the configuration of each virtual object to reflect the weight and form found in each tested reallife object. The weight of each receptacle was approximated to the nearest multiple of $30 \mathrm{~g}$. The objects were chosen to reflect different centers of gravity, as well as their changes.

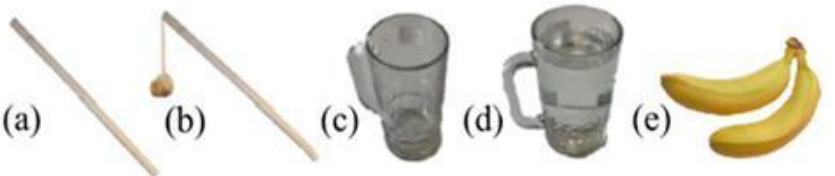

Figure 5: The five everyday objects simulated in this experiment: (a) a rod (RD), (b) a rod with a stone hanging (RS), (c) a cup (GE), (d) a cup full of water $(\mathrm{GF})$, and (e) a pair of bananas $(\mathrm{BN})$.

4.5.1 Results and Discussion. In this experiment, the stacked graph revealed that the accuracy was similarly high for all the virtual objects. However, the "empty" virtual objects were more easily identified as themselves, whereas their fuller counterparts received more precise identifications. Our results indicate that $\mathrm{BN}$ was selected by the participants as a placeholder for the heavier virtual objects because not only was it always correctly identified when presented, it was also selected in GF and RS. Based on the previous results, it was not surprising that the GE and GF were mixed up, given that the most considerable confusion in the previous experiment was also for the Back region (see Figure 6).

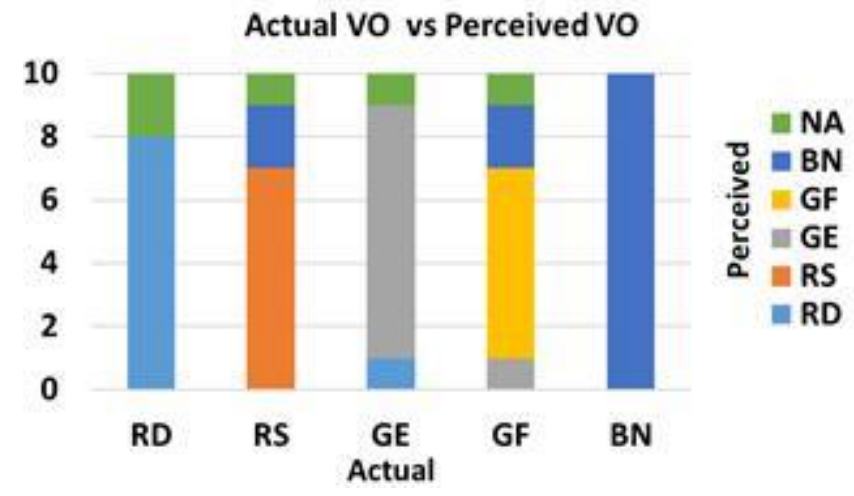

Figure 6: The stacked graph of the comparison between what virtual object was presented to the participants compared to what they perceive them to be. 


\subsection{Evaluation in a VR Application}

We developed a VR fishing game in-house to test how participants would feel using the prototype based on the final design when it is associated to a virtual environment.

Fishing involves adding a mass to the rod's end, which would change the weight and center of gravity. We used Unity3D to create an immersive fishing environment (see Figure 1, most right). The fish were caught randomly (within 1 and 2 minutes) after the player started fishing. When a fish was caught, the weight changed in the front region. In this experiment, participants were presented with the complete device. They were required to put on the backpack and interact with the fishing environment (see Figure 1). After using the VE, they were asked a few questions (see Table 2). A researcher was present to set up the equipment, collect the answers, and respond to any questions that participants had. This was the first time that participants had to wear a VR head-mounted display.

Table 2: Questions asked from participants about the device and experience.

\begin{tabular}{ll|ll}
\hline ID & Question & ID & Question \\
\hline Q1 & Did you feel like you were holding a fishing rod? & Q3 & Could you feel you caught the fish? \\
Q2 & Did you feel like you were fishing? & Q4 & What are your thoughts about the device? \\
\hline
\end{tabular}

4.6.1 Results and Discussion. All participants answered positively to Q2. 9 of the 10 participants also answered positively to Q1. The other participant answered that even though he felt the weight (and center of gravity change), he did not associate it with a fishing rod. Just $20 \%$ of the participants answered negatively to Q3. One of the two participants who had trouble distinguishing the fish/no fish situation was the same one who had trouble distinguishing between the fishing rod and the other objects.

On their general thoughts (Q4), two participants considered the backpack "just a little but not" heavy, and both participants' hands were a "little tired". Nevertheless, all participants declared feeling "very satisfied" after experiencing the VE and the prototype and wanted to add it to other VR applications, as they thought it would be helpful to feel virtual objects in the VE. These results bring a positive light to the prototype and show that most participants can associate the virtual objects with their haptic simulation.

One limitation of the current system is that the final configuration is still not felt instantaneously; however, this limitation is common to most [2, 24, 30], if not all, similar systems. Furthermore, our system is still faster than these other systems, with most people feeling it as satisfactorily fast. Currently, the change is in one dimension, limiting the range of possible objects that can be simulated; however, it still has one more dimension than GravityCup and is faster than Shifty (128 seconds vs. less than 10 seconds). Finally, the extra layer of immersion of haptic sensations is appreciated by all participants who enjoyed having the haptic feeling.

\subsection{Summary of contributions}

In summary, our main contribution is the design process of a prototype that can simulate the weight of everyday objects in VR and their changes in center of gravity. The device has the following features: (1) it can change its mass, (2) it is relatively silent and fast, (3) it can change its center of gravity in $1 \mathrm{D},(4)$ its materials are cheap and easily accessible, and (5) it can be integrated with current VR technologies. The results of the final experiment show its effectiveness in a simulated fishing gaming application. In this game, a change in the weight of the rod represents fish getting caught. This can be emulated in other similar gaming scenarios.

From the results from the five user studies, we can extrapolate the following four human and technical factors that are important to consider: (1) When holding a controller, humans are insensitive to minor variations in weight; thus, precision below a particular value will not necessarily result in better feedback - for an adapted Oculus Touch controller, this value was around $20 \mathrm{~g}$. (2) When limited resources are available, changes in weight should focus on increasing the mass further away from the hand; this will create a fulcrum (lever) effect, making the sensed weight feel heavier and more noticeable. (3) When dealing with changes in weight simulations of long objects, they are easier to be perceived and associated than bulky compact objects. Lastly, (4) A $25 \mathrm{~g} / \mathrm{s}$ change is not fast enough to be imperceptible or to be well-regarded in a gaming application, but at $66 \mathrm{~g} / \mathrm{s}$, it starts to become acceptable. These findings are summarized in Table 3

Table 3: Summary of findings from each experiment

\begin{tabular}{cl}
\hline Experiment & Finding \\
\hline $\mathrm{A}$ & When attached to an Oculus controller changes below $20 \mathrm{~g}$ are indifferent \\
$\mathrm{B}$ & When resources are limited, focus on the mass further away from the hand \\
$\mathrm{C}$ & When attached to an Oculus controller changes of at least $66 \mathrm{~g} / \mathrm{s}$ are necessary \\
$\mathrm{D}$ & Simulations of long objects are easier to be perceived than bulky ones \\
\hline
\end{tabular}

4.7.1 Limitations. Our research presents the final design of a low-cost device with reliable and accurate performance. Though it would be interesting to compare our device with other similar ones, it is challenging to replicate the same device(s) and scenarios used by other researchers. Future research could involve a comparative study involving our device and other similar devices to assess their relative performance and usability and to gain further insights into the development of low-cost devices that can simulate the weight of virtual objects and changes in their center of gravity.

Any electronic system communication can potentially have transmission delays. However, we did not calculate this delay because the time for communication was of a smaller magnitude than the the used for the system to be filled. Also, our system was faster than other reported state-of-the art systems (e.g., [18]). In our last experiment, We did not measure the time from when the participants saw the event in VR until feeling the corresponding weight, as participants said that they felt the weight change 'almost' instantaneously. As such, the latency, if any, was not noticeable by participants and not an issue for our prototype. Similarly, because we have used an open system, there might have been an additive error after each experiment. However, the threaded rod system presented an error of $1 \mathrm{~g}$ after 60 activations, which represented a lower number of activations experienced by the participants and a lower threshold of what participants declared being able to dif-ferentiate. Moreover, the pump system was only used on the back part of the balloon, where small variations were not strongly felt. As such, additive errors, if any, did not represent an issue for the overall functioning of the system. On the other hand, a simple way to address any such errors is to set a automatic reset after a certain number of activations.

In our experiments, the perception was based on a single sample from each participant. We did this because we observed in pilot studies that, after a few repetitions of the same trials, fatigue could kick in and could affect their responses. However, the results we had were consistent among themselves, showing errors were committed by a similar number of participants, and independent participants often chose similar answers. As such, to a large extent, our approach is valid, as the performance of the final complete prototype linked to the VR application shows. 


\section{CONCLUSION}

This research presented the design and development process of a low-cost device for weight and center of gravity simulation for virtual reality (VR) applications based on fluid relocation. We presented a series of user studies that allowed us to understand the requirements needed to develop a haptic weight device that can be attached to existing VR controllers. We learned that (1) when adding the device to the existing controller, there is no need for precision more significant than $20 \mathrm{~g}$, (2) up to $200 \mathrm{~g}$ the just-noticeable difference is similar for his kind of application, (3) because it works as a fulcrum, weights further from the controller are easier to perceive, and (4) speeds of $25 \mathrm{~g} / \mathrm{s}$ are not enough to give users satisfactory haptic feedback. Finally, after studying three ways to transport fluid from a backpack reservoir to a holder attached to the hand-held controller, a mixed model has been used to develop the device to simulate up to $500 \mathrm{~g}$ using both pump system and threaded rod methods. The pump system allows fast transfer while the threaded rod gives precise sensations to the users. As such, this combination allows the weight to be distributed efficiently and lets users have an accurate haptic sensation.

Our design allows the device to be attached to a VR handheld controller, like the Oculus Touch, without needing any other particular adaptations. Our experiments and results can play a valuable role for future work that aims to develop additive technologies for current VR devices because they provide a baseline for (1) the different weights participants can detect; (2) where weight changes are easier to be detected; and (3) how fast or slow a system should be to be acceptable by users. This technology can be used in VR training applications so that trainees can better grasp the tools they are using. It can also be used in games so that a player can feel the items they are holding, like weapons in first-person shooter games or weights in exergames. Its portability follows the current trend of making VR devices that are more and more mobile (like the Oculus Quest).

\section{ACKNOWLEDGMENTS}

The authors thank the participants for their time and the reviewers for their insightful comments that have helped improve our paper.

\section{REFERENCES}

[1] Merwan Achibet, Adrien Girard, Anthony Talvas, Maud Marchal, and Anatole Lécuyer. 2015. Elastic-Arm: Human-scale passive haptic feedback for augmenting interaction and perception in virtual environments. In 2015 IEEE Virtual Reality (VR). IEEE, 63-68.

[2] Chih-Hao Cheng, Chia-Chi Chang, Ying-Hsuan Chen, Ying-Li Lin, Jing-Yuan Huang, Ping-Hsuan Han, Ju-Chun Ko, and Lai-Chung Lee. 2018. GravityCup: a liquid-based haptics for simulating dynamic weight in virtual reality. In Pro-ceedings of the 24th ACM Symposium on Virtual Reality Software and Technology. 1-2.

[3] Inrak Choi, Heather Culbertson, Mark R Miller, Alex Olwal, and Sean Follmer. 2017. Grabity: A wearable haptic interface for simulating weight and grasping in virtual reality. In Proceedings of the 30th Annual ACM Symposium on User Interface Software and Technology. 119-130.

[4] Lionel Dominjon, Anatole Lécuyer, J-M Burkhardt, Paul Richard, and Simon Richir. 2005. Influence of control/display ratio on the perception of mass of manipulated objects in virtual environments. In IEEE Proceedings. VR 2005. Virtual Reality, 2005. IEEE, 19-25.

[5] Hakan B Gurocak and Benjamin Parrish. 2002. AirGlove: a force feedback device for virtual reality. In Telemanipulator and Telepresence Technologies VIII, Vol. 4570. International Society for Optics and Photonics, 69-77.

[6] Teng Han, Fraser Anderson, Pourang Irani, and Tovi Grossman. 2018. Hydroring: Supporting mixed reality haptics using liquid flow. In
Proceedings of the 31st Annual ACM Symposium on User Interface Software and Technology. 913-925.

[7] Seongkook Heo, Christina Chung, Geehyuk Lee, and Daniel Wigdor. 2018. Thor's hammer: An ungrounded force feedback device utilizing propeller-induced propulsive force. In Proceedings of the $2018 \mathrm{CHI}$ Conference on Human Factors in Computing Systems. 1-11.

[8] Paul Issartel, Florimond Guéniat, Sabine Coquillart, and Mehdi Ammi. 2015. Perceiving mass in mixed reality through pseudo-haptic rendering of Newton's third law. In 2015 IEEE Virtual Reality (VR). IEEE, 41-46.

[9] David Antonio Gómez Jáuregui, Ferran Argelaguet, Anne-Hélène Olivier, Maud Marchal, Franck Multon, and Anatole Lecuyer. 2014. Toward" pseudo-haptic avatars": Modifying the visual animation of self-avatar can simulate the percep-tion of weight lifting. IEEE transactions on visualization and computer graphics 20, 4 (2014), 654-661.

[10] Seungwoo Je, Myung Jin Kim, Woojin Lee, Byungjoo Lee, XingDong Yang, Pedro Lopes, and Andrea Bianchi. 2019. Aero-plane: A handheld force-feedback device that renders weight motion illusion on a virtual $2 \mathrm{~d}$ plane. In Proceedings of the 32nd Annual ACM Symposium on User Interface Software and Technology. 763-775.

[11] Anatole Lécuyer. 2009. Simulating haptic feedback using vision: A survey of research and applications of pseudo-haptic feedback. Presence: Teleoperators and Virtual Environments 18, 1 (2009), 39-53.

[12] Ming C Lin and Miguel Otaduy. 2008. Haptic rendering: foundations, algorithms, and applications. CRC Press.

[13] Lili Liu, Rachael Ip, Anna Shum, and Christian Wagner. 2014. Learning effects of virtual game worlds: an empirical investigation of immersion, enjoyment and performance. (2014).

[14] John C McClelland, Robert J Teather, and Audrey Girouard. 2017. Haptobend: shape-changing passive haptic feedback in virtual reality. In Proceedings of the 5th Symposium on Spatial User Interaction. 8290.

[15] Kouta Minamizawa, Souichiro Fukamachi, Hiroyuki Kajimoto, Naoki Kawakami, and Susumu Tachi. 2007. Gravity grabber: wearable haptic display to present virtual mass sensation. In ACM SIGGRAPH 2007 emerging technologies. 8-es.

[16] Ryuma Niiyama, Lining Yao, and Hiroshi Ishii. 2014. Weight and volume changing device with liquid metal transfer. In Proceedings of the 8th International Conference on Tangible, Embedded and Embodied Interaction. 49-52.

[17] Hossein Noureddini, BC Teoh, and L Davis Clements. 1992. Viscosities of veg-etable oils and fatty acids. Journal of the American Oil Chemists' Society 69, 12 (1992), 1189-1191.

[18] Chung Hyuk Park, Kenneth L Wilson, and Ayanna M Howard. 2013. Examining the learning effects of a low-cost haptic-based virtual reality simulator on laparo-scopic cholecystectomy. In Proceedings of the 26th IEEE international symposium on computer-based medical systems. IEEE, 233-238.

[19] Jotaro Shigeyama, Takeru Hashimoto, Shigeo Yoshida, Takuji Narumi, Tomohiro Tanikawa, and Michitaka Hirose. 2019. Transcalibur: A weight shifting virtual reality controller for $2 \mathrm{~d}$ shape rendering based on computational perception model. In Proceedings of the 2019 CHI Conference on Human Factors in Computing Systems. 1-11.

[20] Tadeusz Stach and TC Nicholas Graham. 2011. Exploring haptic feedback in exergames. In IFIP conference on human-computer interaction. Springer, 18-35.

[21] Evan Strasnick, Christian Holz, Eyal Ofek, Mike Sinclair, and Hrvoje Benko. 2018. Haptic links: Bimanual haptics for virtual reality using variable stiffness actuation. In Proceedings of the $2018 \mathrm{CHI}$ Conference on Human Factors in Computing Systems. 1-12.

[22] Colin Swindells, Alex Unden, and Tao Sang. 2003. TorqueBAR: an ungrounded haptic feedback device. In Proceedings of the 5th international conference on Mul-timodal interfaces. 52-59. 
[23] Vânia Regina Nicoletti Telis, Javier Telis-Romero, HB Mazzotti, and Ana Lúcia Gabas. 2007. Viscosity of aqueous carbohydrate solutions at different temper-atures and concentrations. International Journal of food properties 10, 1 (2007), 185-195.

[24] Shan-Yuan Teng, Tzu-Sheng Kuo, Chi Wang, Chi-huan Chiang, DaYuan Huang, Liwei Chan, and Bing-Yu Chen. 2018. Pupop: Pop-up prop on palm for virtual re-ality. In Proceedings of the 31st Annual ACM Symposium on User Interface Software and Technology. 5-17.

[25] Julie M Walker, Heather Culbertson, Michael Raitor, and Allison M Okamura. 2017. Haptic orientation guidance using two parallel double-gimbal control moment gyroscopes. IEEE transactions on haptics 11, 2 (2017), 267-278.

[26] Eric Whitmire, Hrvoje Benko, Christian Holz, Eyal Ofek, and Mike Sinclair. 2018. Haptic revolver: Touch, shear, texture, and shape rendering on a reconfigurable virtual reality controller. In Proceedings of the 2018 CHI Conference on Human Factors in Computing Systems. 1-12.

[27] Kyle N Winfree, Joseph M Romano, Jamie Gewirtz, and Katherine J Kuchenbecker. 2010. Control of a high fidelity ungrounded torque feedback device: The iTorqU 2.1. In 2010 IEEE International Conference on Robotics and Automation. IEEE, 1347-1352.

[28] Nick Yee. 2006. The demographics, motivations and derived experiences of users of massively-multiuser online graphical environments. PRESENCE: Virtual and Augmented Reality, 15 (3), 309-329.

[29] Run Yu and Doug A Bowman. 2020. Pseudo-haptic display of mass and mass distri-bution during object rotation in virtual reality. IEEE transactions on visualization and computer graphics 26, 5 (2020), 2094-2103.

[30] Andre Zenner and Antonio Krüger. 2017. Shifty: A weight-shifting dynamic pas-sive haptic proxy to enhance object perception in virtual reality. IEEE transactions on visualization and computer graphics 23 , 4 (2017), 1285-1294.

\section{A APPENDIX}

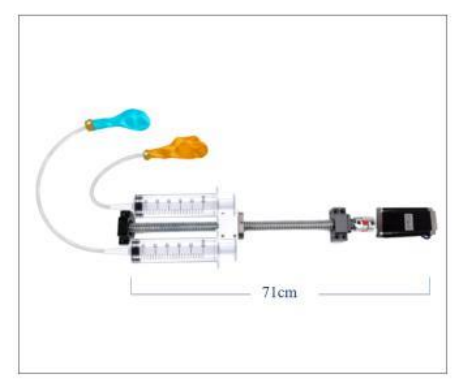

(1) Needle tubing-based System

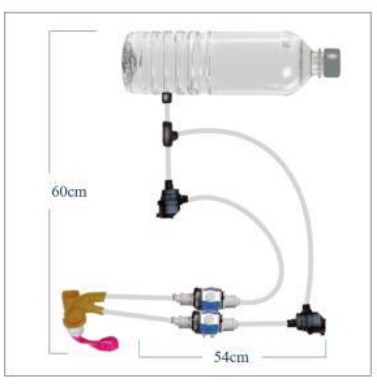

(2) Pump based Equipment
Figure 7: Two diagrams showing the connection of all the components of the pump-based weight simulation system.

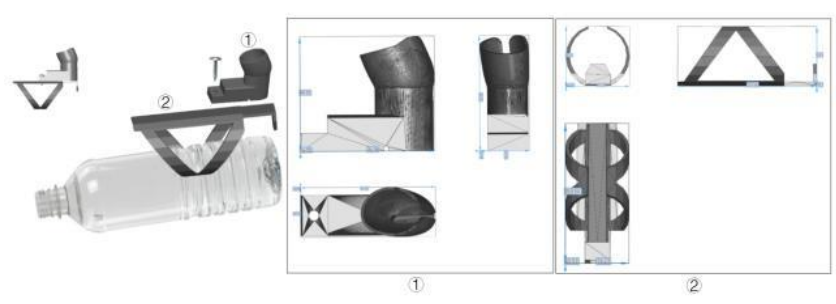

Figure 8: A diagram with the schematics of the 3D printed unit that allows attaching the bottle receptacle containing the balloons to a VR handheld controller, like the Oculus Touch. (Left) An overview of all the parts and how they fit together; (1): Three views of the $3 \mathrm{D}$ printed parts that connect to the handheld controller; (2): Three views of the parts that connect to the plastic bottle containing the balloons. 